\title{
SOLUTION OF SEMICOERCIVE SIGNORINI PROBLEM BASED ON A DUALITY SCHEME WITH MODIFIED LAGRANGIAN FUNCTIONAL
}

\author{
Robert V. Namm, Gyungsoo Woo*, Shu-Sen Xie, and Sucheol Yi
}

\begin{abstract}
In this paper, the iterative Uzawa method with a modified Lagrangian functional is investigated to seek a saddle point for the semicoercive variational Signorini inequality.
\end{abstract}

\section{Introduction}

The construction and the analysis of duality methods for solving elliptic variational inequalities in mechanics are usually based on classical duality schemes. Therefore, it is assumed that the functionals to be minimized are strongly convex. However, for a number of practically important variational inequalities, strong convexity holds only on finite codimensional subspaces of the original Hilbert space. The use of the classical Lagrangian functional in such semicoercive problems does not guarantee the convergence of the available methods for finding saddle points $([5,4])$, and, hence, a modified Lagrangian functional $([9,8])$ may be more appropriate to use. In this paper, the Uzawa method ([1]) with a modified Lagrangian functional is investigated for an application to find a saddle point in the scalar semicoercive Signorini problem.

\section{Modified Lagrangian functional}

Consider the following variational Signorini problem (see $[3,6])$

$$
\left\{\begin{array}{l}
J(v)=\frac{1}{2} \int|\nabla v|^{2}-\int f v-\min \\
v \in \mathcal{K}=\left\{w \in W_{2}^{1}(\Omega): \gamma v \geq 0 \text { a.e. on } \Gamma\right\}
\end{array}\right.
$$

Here, $\Omega \subset \mathbb{R}^{n}(n=2,3)$ is a bounded domain with a sufficiently smooth boundary $\Gamma, f \in L_{2}(\Omega)$ is a given function, and $\gamma w \in W_{2}^{1 / 2}(\Gamma)$ is the trace of $w \in W_{2}^{1}(\Omega)$ on $\Gamma$.

Received June 2, 2011.

2010 Mathematics Subject Classification. Primary 65F10, 65K10, 49M15, 74G15, 74G65. Key words and phrases. Signorini problem, modified Lagrangian functional, saddle point, Uzawa method, Gâteaux derivative.

* The work of this author was supported by National Research Foundation Grant 20090077297 and in part by Changwon National University in 2009. 
If the solution $u$ to problem (1) belongs to the space $W_{2}^{2}(\Omega)$, then $u$ satisfies the nonlinear boundary value problem (see [3])

$$
\left\{\begin{array}{l}
-\Delta u=f \text { in } \Omega, \\
u \geq 0, \frac{\partial u}{\partial n} \geq 0, u \frac{\partial u}{\partial n}=0 \text { on } \Gamma,
\end{array}\right.
$$

where $n$ is the unit outer normal to $\Gamma$. Since the functional $J(v)$ is not strictly coercive on $\Gamma$, problem (1) may have no solutions. However, if

$$
\int_{\Omega} f d \Omega<0
$$

then $J(v) \rightarrow+\infty$ as $\|v\|_{W_{2}^{1}(\Omega)} \rightarrow \infty, v \in \mathcal{K}$, and, hence, problem (1) is solvable (cf. $[3,2])$. Moreover, in view of $(3)$, the solution is unique. In what follows, we assume that condition (3) is fulfilled.

Consider the classical Lagrangian functional

$$
\begin{aligned}
& L(v, l) \\
= & J(v)-\int_{\Gamma} l \gamma v d \Gamma \\
(5)= & \frac{1}{2} \int_{\Omega}|\nabla v|^{2} d \Omega-\int_{\Omega} f v d \Omega-\int_{\Gamma} l \gamma v d \Gamma \quad(v, l) \in W_{2}^{1}(\Omega) \times L_{2}(\Gamma) .
\end{aligned}
$$

Let $\left(L_{2}(\Gamma)\right)^{+}$be the cone of square integrable functions on $\Gamma$.

Definition. A point $\left(v^{*}, l^{*}\right) \in W_{2}^{1}(\Omega) \times\left(L_{2}(\Gamma)\right)^{+}$is called a saddle point of the classical Lagrangian functional $L(v, l)$ if

$$
L\left(v^{*}, l\right) \leq L\left(v^{*}, l^{*}\right) \leq L\left(v, l^{*}\right) \quad \forall(v, l) \in W_{2}^{1}(\Omega) \times\left(L_{2}(\Gamma)\right)^{+} .
$$

It has been shown in [9] that if the solution $u$ of problem (1) belongs to the space $W_{2}^{2}(\Omega)$, then the Lagrangian functional has the unique saddle point $\left(u, \frac{\partial u}{\partial n}\right)$; i.e., $v^{*}=u$ almost everywhere in $\Omega$ and $l^{*}=\frac{\partial u}{\partial n}$ almost everywhere on $\Gamma$.

Nevertheless, an application of the Uzawa method to the search for a saddle point of $L(v, l)$ is impossible in the semicoercive case, because the convergence of this iterative method is ensured by coordinating the step size for the dual variable $l$ with the positive definiteness constant in the quadratic form of the functional to be minimized. However, in semicoercive problem (1), the quadratic form

$$
a(v, v)=\int_{\Omega}|\nabla v|^{2} d \Omega
$$

is only positive semidefinite. To overcome this difficulty, consider a modification of the Lagrangian functional.

We define the following functional on the space $W_{2}^{1}(\Omega) \times L_{2}(\Gamma) \times L_{2}(\Gamma)$ :

$$
\mathrm{K}(v, l, m)= \begin{cases}J(v)+\frac{1}{2 r} \int_{\Gamma}\left((l+r m)^{2}-l^{2}\right) d \Gamma \quad \text { if }-\gamma v \leq m \text { a.e. on } \Gamma \\ +\infty \quad \text { otherwise, }\end{cases}
$$


where $r>0$ is a constant. It can be easily seen that

$$
\begin{aligned}
\inf _{m} K(v, l, m) & =\inf _{-\gamma v \leq m}\left\{J(v)+\frac{1}{2 r} \int_{\Gamma}\left((l+r m)^{2}-l^{2}\right) d \Gamma\right\} \\
& =J(v)+\frac{1}{2 r} \inf _{-\gamma v \leq m} \int_{\Gamma}\left((l+r m)^{2}-l^{2}\right) d \Gamma \\
& =J(v)+\frac{1}{2 r} \int_{\Gamma}\left(\left((l-r \gamma v)^{+}\right)^{2}-l^{2}\right) d \Gamma
\end{aligned}
$$

where $(l-r \gamma v)^{+}=\max \{0, l-r \gamma v\}$.

We define the modified Lagrangian functional $M(v, l)$ on the space $W_{2}^{1}(\Omega) \times$ $L_{2}(\Gamma)$ as

(6) $\mathrm{M}(v, l)=\inf _{m} \mathrm{~K}(v, l, m)=J(v)+\frac{1}{2 r} \int_{\Gamma}\left(\left((l-r \gamma v)^{+}\right)^{2}-l^{2}\right) d \Gamma$.

It can be shown that the functional $M(v, l)$ is convex with respect to $v$ for a fixed $l$ and is concave with respect to $l$ for a fixed $v$. Moreover, $M(v, l)$ is Gâteaux differentiable in both its variables $v$ and $l$. Furthermore, the Gâteaux derivatives $\nabla_{v} M(v, l)$ and $\nabla_{l} M(v, l)$ satisfy the equalities

$$
\begin{aligned}
& \left(\nabla_{v} M(v, l), h\right)=a(v, h)-(f, h)-\int_{\Gamma}(l-r \gamma v)^{+} h d \Gamma \quad \forall h \in W_{2}^{1}(\Omega), \\
& \left(\nabla_{l} M(v, l), \theta\right)=\frac{1}{r} \int_{\Gamma}\left((l-r \gamma v)^{+}-l\right) \theta d \Gamma \quad \forall \theta \in L_{2}(\Gamma),
\end{aligned}
$$

where

$$
a(v, h)=\int_{\Omega} \nabla v \nabla h d \Omega, \quad(f, h)=\int_{\Omega} f h d \Omega .
$$

Definition. A point $\left(v^{*}, l^{*}\right) \in W_{2}^{1}(\Omega) \times L_{2}(\Gamma)$ is called a saddle point of the modified Lagrangian functional $M(v, l)$ if

$$
\mathrm{M}\left(v^{*}, l\right) \leq \mathrm{M}\left(v^{*}, l^{*}\right) \leq \mathrm{M}\left(v, l^{*}\right) \quad \forall(v, l) \in W_{2}^{1}(\Omega) \times L_{2}(\Gamma) .
$$

Let us define the functional

$$
\underline{\mathrm{M}}(l)=\inf _{v} \mathrm{M}(v, l)=\inf _{v} \inf _{m} \mathrm{~K}(v, l, m) .
$$

Since

$$
\inf _{v} \inf _{m} \mathrm{~K}(v, l, m)=\inf _{m} \inf _{v} \mathrm{~K}(v, l, m),
$$

we get

$$
\begin{aligned}
\underline{\mathrm{M}}(l) & =\inf _{m} \inf _{v} \mathrm{~K}(v, l, m) \\
& =\inf _{m} \inf _{-\gamma v \leq m}\left\{J(v)+\frac{1}{2 r} \int_{\Gamma}\left((l+r m)^{2}-l^{2}\right) d \Gamma\right\} \\
& =\inf _{m}\left\{\inf _{-\gamma v \leq m} J(v)+\int_{\Gamma} l m d \Gamma+\frac{r}{2} \int_{\Gamma} m^{2} d \Gamma\right\}
\end{aligned}
$$




$$
=\inf _{m}\left\{\chi(m)+\int_{\Gamma} l m d \Gamma+\frac{r}{2} \int_{\Gamma} m^{2} d \Gamma\right\},
$$

where $\chi(m)=\inf _{-\gamma v<m} J(v)$.

It is easy to show that $\chi(m)$ is a convex function (see $[9,1])$ and $\chi(m) \neq-\infty$ for all $m \in L_{2}(\Gamma)$. Thus, the functional $\underline{\mathrm{M}}(l)$ has the following two representations:

$$
\begin{gathered}
\underline{\mathrm{M}}(l)=\inf _{v}\left\{J(v)+\frac{1}{2 r} \int_{\Gamma}\left(\left((l-r \gamma v)^{+}\right)^{2}-l^{2}\right) d \Gamma\right\}, \\
\underline{\mathrm{M}}(l)=\inf _{m}\left\{\chi(m)+\int_{\Gamma} l m d \Gamma+\frac{r}{2} \int_{\Gamma} m^{2} d \Gamma\right\} .
\end{gathered}
$$

Since the expression $\chi(m)+\int_{\Gamma} l m d \Gamma+\frac{r}{2} \int_{\Gamma} m^{2} d \Gamma$ is a strongly convex functional with respect to $m \in L_{2}(\Gamma)$, the infimum in problem (9) is attained at a unique element $m(l)$ for an arbitrary fixed $l \in L_{2}(\Gamma)$.

Theorem 2.1. Problem (8) is solvable for an arbitrary fixed $l \in L_{2}(\Gamma)$.

Proof. Since the problem

$$
\left\{\begin{array}{l}
J(v)-\min \\
-\gamma v \leq m
\end{array}\right.
$$

is solvable under arbitrary $m \in L_{2}(\Gamma)$ (cf. [1]), we can define

$$
v(l)=\arg \min _{-\gamma v \leq m(l)} J(v) \quad \forall l \in L_{2}(\Gamma) .
$$

We then have

$$
\underline{\mathrm{M}}(l)=J(v(l))+\int_{\Gamma} l m(l) d \Gamma+\frac{r}{2} \int_{\Gamma}(m(l))^{2} d \Gamma=\mathrm{K}(v(l), l, m(l)) .
$$

From (3), we get

$$
\begin{aligned}
& \inf _{v}\left\{J(v)+\frac{1}{2 r} \inf _{-\gamma v \leq m} \int_{\Gamma}\left((l+r m)^{2}-l^{2}\right) d \Gamma\right\} \\
= & \inf _{v}\left\{J(v)+\frac{1}{2 r} \int_{\Gamma}\left(\left((l-r \gamma v)^{+}\right)^{2}-l^{2}\right) d \Gamma\right\} \\
= & \underline{\mathrm{M}}(l)=\inf _{v} \inf _{m} \mathrm{~K}(v, l, m)=\inf _{m} \inf _{v} \mathrm{~K}(v, l, m) \\
= & J(v(l))+\frac{1}{2 r} \int_{\Gamma}\left((l+r m(l))^{2}-l^{2}\right) d \Gamma .
\end{aligned}
$$

We will now show that

$J(v(l))+\frac{1}{2 r} \int_{\Gamma}\left((l+r m(l))^{2}-l^{2}\right) d \Gamma=J(v(l))+\frac{1}{2 r} \inf _{-\gamma v(l) \leq m} \int_{\Gamma}\left((l+r m)^{2}-l^{2}\right) d \Gamma$.

For our proof we will use a contradiction. Suppose we had

$J(v(l))+\frac{1}{2 r} \int_{\Gamma}\left((l+r m(l))^{2}-l^{2}\right) d \Gamma>J(v(l))+\frac{1}{2 r} \inf _{-\gamma v(l) \leq m} \int_{\Gamma}\left((l+r m)^{2}-l^{2}\right) d \Gamma ;$ 
that is,

$$
\int_{\Gamma}\left((l+r m(l))^{2}-l^{2}\right) d \Gamma>\inf _{-\gamma v(l) \leq m} \int_{\Gamma}\left((l+r m)^{2}-l^{2}\right) d \Gamma .
$$

We then have

$$
\begin{aligned}
& \inf _{v}\left\{J(v)+\frac{1}{2 r} \inf _{-\gamma v \leq m} \int_{\Gamma}\left((l+r m)^{2}-l^{2}\right) d \Gamma\right\} \\
= & \underline{\mathrm{M}}(l)=J(v(l))+\frac{1}{2 r} \int_{\Gamma}\left((l+r m(l))^{2}-l^{2}\right) d \Gamma \\
> & J(v(l))+\frac{1}{2 r} \inf _{-\gamma v(l) \leq m} \int_{\Gamma}\left((l+r m)^{2}-l^{2}\right) d \Gamma \\
\geq & \inf _{v}\left\{J(v)+\frac{1}{2 r} \inf _{-\gamma v \leq m} \int_{\Gamma}\left((l+r m)^{2}-l^{2}\right) d \Gamma\right\},
\end{aligned}
$$

which is a contradiction, and, hence, (11) holds.

It follows from (10) and (11) that problem (8) is solvable and

$$
v(l)=\arg \min _{v}\left\{J(v)+\frac{1}{2 r} \int_{\Gamma}\left(\left((l-r \gamma v)^{+}\right)^{2}-l^{2}\right) d \Gamma\right\} .
$$

Remark 2.2. Theorem 2.1 was proved in [1] for $l \in\left(L_{2}(\Gamma)\right)^{+}$only.

Consider the problem

$$
\left\{\begin{array}{l}
\underline{\mathrm{M}}(l)-\max \\
l \in L_{2}(\Gamma)
\end{array}\right.
$$

which is called the dual of problem (1).

Let us define the functional

$$
\overline{\mathrm{M}}(v)=\sup _{l \in L_{2}(\Gamma)} \mathrm{M}(v, l) \quad \forall v \in W_{2}^{1}(\Omega) .
$$

Suppose that $v \in G$. Then $\mathrm{K}(v, l, 0)=J(v)$ for all $l \in L_{2}(\Gamma)$, and, thus, we have

$$
\mathrm{M}(v, l)=\inf _{m \in L_{2}(\Gamma)} \mathrm{K}(v, l, m) \leq J(v) \quad \forall v \in \mathcal{K} .
$$

Hence, we get

$$
\overline{\mathrm{M}}(v)=\sup _{l \in L_{2}(\Gamma)} \mathrm{M}(v, l) \leq J(v) \quad \forall v \in \mathcal{K} .
$$

If $-\gamma v \leq m$ a.e. on $\Gamma$, then we have

$$
\mathrm{K}(v, 0, m)=J(v)+\frac{r}{2} \int_{\Gamma} m^{2} d \Gamma .
$$

Therefore, we obtain

$$
\begin{aligned}
\mathrm{M}(v, 0) & =\inf _{m \in L_{2}(\Gamma)} \mathrm{K}(v, 0, m)=\inf _{-\gamma v \leq m} \mathrm{~K}(v, 0, m) \\
& =\inf _{-\gamma v \leq m}\left\{J(v)+\frac{r}{2} \int_{\Gamma} m^{2} d \Gamma\right\}
\end{aligned}
$$




$$
=J(v)+\frac{r}{2} \inf _{-\gamma v \leq m} \int_{\Gamma} m^{2} d \Gamma \geq J(v) \quad \forall v \in W_{2}^{1}(\Omega) .
$$

We then have

$$
\overline{\mathrm{M}}(v)=\sup _{l \in L_{2}(\Gamma)} \mathrm{M}(v, l) \geq \mathrm{M}(v, 0) \geq J(v) \quad \forall v \in W_{2}^{1}(\Omega) .
$$

It follows from (13) and (14) that

$$
\overline{\mathrm{M}}(v)=J(v) \quad \forall v \in \mathcal{K} .
$$

We now consider the functional

$$
\overline{\mathrm{K}}(v, l, m)= \begin{cases}J(v)+\int_{\Gamma} l m d \Gamma, & \text { if }-\gamma v \leq m \text { a.e. on } \Gamma, \\ +\infty & \text { otherwise. }\end{cases}
$$

One can easily see that

$$
\inf _{m \in L_{2}(\Gamma)} \overline{\mathrm{K}}(v, l, m)=J(v)+\int_{\Gamma} l m d \Gamma=L(v, l) \quad \forall l \in\left(L_{2}(\Gamma)\right)^{+},
$$

where $L(v, l)$ is the quantity appears in (4), and

$$
\mathrm{K}(v, l, m) \geq \overline{\mathrm{K}}(v, l, m)
$$

which implies

$$
\mathrm{M}(v, l) \geq L(v, l) \quad \forall l \in\left(L_{2}(\Gamma)\right)^{+} .
$$

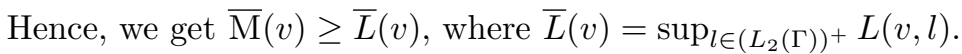

If $v \notin \mathcal{K}$, then

$$
\bar{L}(v)=\sup _{l \in\left(L_{2}(\Gamma)\right)^{+}} L(v, l)=\sup _{l \in\left(L_{2}(\Gamma)\right)^{+}}\left\{J(v)-\int_{\Gamma} l \gamma v d \Gamma\right\}=+\infty,
$$

and, thus, $\overline{\mathrm{M}}(v)=+\infty \forall v \notin \mathcal{K}$. From (15) and (16), we have

$$
\overline{\mathrm{M}}(v)= \begin{cases}J(v), & \text { if } v \in \mathcal{K}, \\ +\infty & \text { otherwise. }\end{cases}
$$

Hence, problem (1) can be presented as

$$
\left\{\begin{array}{l}
\overline{\mathrm{M}}(v)-\min \\
v \in W_{2}^{1}(\Omega)
\end{array}\right.
$$

It is obvious that

$$
\begin{gathered}
\inf _{v \in W_{2}^{1}(\Omega)} \sup _{l \in L_{2}(\Gamma)} \mathrm{M}(v, l) \geq \sup _{l \in L_{2}(\Gamma)} \inf _{v \in W_{2}^{1}(\Omega)} \mathrm{M}(v, l), \\
\inf _{v \in W_{2}^{1}(\Omega)} \overline{\mathrm{M}}(v) \geq \sup _{l \in L_{2}(\Gamma)} \underline{\mathrm{M}}(l) .
\end{gathered}
$$

Suppose that we have equality

$$
\overline{\mathrm{M}}\left(v^{*}\right)=\underline{\mathrm{M}}\left(l^{*}\right)
$$

for some $v^{*} \in W_{2}^{1}(\Omega)$ and $l^{*} \in L_{2}(\Gamma)$. Then from (18), we can see that $v^{*}$ and $l^{*}$ are solutions of problems (17) and (12), respectively. 
It is easy to show that $\left(v^{*}, l^{*}\right)$ is a saddle point of the modified Lagrangian functional $M(v, l)$.

Theorem 2.3. In order that $\left(v^{*}, l^{*}\right) \in W_{2}^{1}(\Omega) \times L_{2}(\Gamma)$ is a saddle point of $M(v, l)$ in (6), it is necessary and sufficient for $v^{*}$ to be a solution of Signorini problem (1) and for all $m \in L_{2}(\Gamma)$ the inequality

$$
\chi(m)+\int_{\Gamma} l^{*} m d \Gamma+\frac{r}{2} \int_{\Gamma} m^{2} d \Gamma \geq \chi(0)
$$

must be fulfilled.

Proof. Let $\left(v^{*}, l^{*}\right) \in W_{2}^{1}(\Omega) \times L_{2}(\Gamma)$ be a saddle point of $M(v, l)$. This means that

$$
\sup _{l \in L_{2}(\Gamma)} \mathrm{M}\left(v^{*}, l\right)=\mathrm{M}\left(v^{*}, l^{*}\right)=\inf _{v \in W_{2}^{1}(\Omega)} \mathrm{M}\left(v, l^{*}\right)
$$

$$
\overline{\mathrm{M}}\left(v^{*}\right)=\underline{\mathrm{M}}\left(l^{*}\right),
$$

from which and (18), we can see that $v^{*}$ is a solution of problem (17) and $l^{*}$ is a solution of problem (12), respectively. Hence, we get

$$
\underline{\mathrm{M}}\left(l^{*}\right)=\overline{\mathrm{M}}\left(v^{*}\right)=\min _{v \in \mathcal{K}} J(v)=\chi(0),
$$

which means that

$$
\inf _{m \in L_{2}(\Gamma)}\left\{\chi(m)+\int_{\Gamma} l^{*} m d \Gamma+\frac{r}{2} \int_{\Gamma} m^{2} d \Gamma\right\}=\chi(0)
$$

that is,

$$
\chi(m)+\int_{\Gamma} l^{*} m d \Gamma+\frac{r}{2} \int_{\Gamma} m^{2} d \Gamma \geq \chi(0) \quad \forall m \in L_{2}(\Gamma) .
$$

Let $v^{*}$ be a solution of problem (17) and

$$
\chi(m)+\int_{\Gamma} l^{*} m d \Gamma+\frac{r}{2} \int_{\Gamma} m^{2} d \Gamma \geq \chi(0) \quad \forall m \in L_{2}(\Gamma) .
$$

We then have

$$
\underline{\mathrm{M}}\left(l^{*}\right)=\inf _{m \in L_{2}(\Gamma)}\left\{\chi(m)+\int_{\Gamma} l^{*} m d \Gamma+\frac{r}{2} \int_{\Gamma} m^{2} d \Gamma\right\} \geq \chi(0)=\overline{\mathrm{M}}\left(v^{*}\right) .
$$

Since $\overline{\mathrm{M}}(v) \geq \underline{\mathrm{M}}(l) \quad \forall(v, l) \in W_{2}^{1}(\Omega) \times L_{2}(\Gamma)$, we get

$$
\overline{\mathrm{M}}\left(v^{*}\right)=\underline{\mathrm{M}}\left(l^{*}\right) \text {. }
$$

It is obvious that

$$
\inf _{v \in W_{2}^{1}(\Omega)} M\left(v, l^{*}\right) \leq M\left(v^{*}, l^{*}\right) \leq \sup _{l \in L_{2}(\Gamma)} M\left(v^{*}, l\right) .
$$

Therefore, we obtain

$$
\inf _{v \in W_{2}^{1}(\Omega)} M\left(v, l^{*}\right)=M\left(v^{*}, l^{*}\right)=\sup _{l \in L_{2}(\Gamma)} M\left(v^{*}, l\right) ;
$$


that is,

$$
M\left(v^{*}, l\right) \leq M\left(v^{*}, l^{*}\right) \leq M\left(v, l^{*}\right) \quad \forall(v, l) \in W_{2}^{1}(\Omega) \times L_{2}(\Gamma)
$$

Theorem 2.4. Let $\left(v^{*}, l^{*}\right) \in W_{2}^{1}(\Omega) \times\left(L_{2}(\Gamma)\right)^{+}$be a saddle point of the classical Lagrangian functional $L(v, l)$ given in $(4)$. Then $\left(v^{*}, l^{*}\right)$ is a saddle point of modified Lagrangian functional $M(v, l)$ in $(6)$.

Proof. We have

$$
L\left(v^{*}, l\right) \leq L\left(v^{*}, l^{*}\right) \leq L\left(v, l^{*}\right) \quad \forall(v, l) \in W_{2}^{1}(\Omega) \times\left(L_{2}(\Gamma)\right)^{+} .
$$

This means

$$
\begin{aligned}
\sup _{l \in\left(L_{2}(\Gamma)\right)^{+}} L\left(v^{*}, l\right) & =L\left(v^{*}, l^{*}\right)=\inf _{v \in W_{2}^{1}(\Omega)} L\left(v, l^{*}\right), \\
\bar{L}\left(v^{*}\right) & =L\left(v^{*}, l^{*}\right)=\underline{L}\left(l^{*}\right),
\end{aligned}
$$

where

$$
\begin{aligned}
& \bar{L}(v)=\sup _{l \in\left(L_{2}(\Gamma)\right)^{+}} L(v, l) \quad \forall v \in W_{2}^{1}(\Omega), \\
& \underline{L}(l)=\inf _{v \in W_{2}^{1}(\Omega)} L(v, l) \quad \forall l \in\left(L_{2}(\Gamma)\right)^{+} .
\end{aligned}
$$

Taking into account that $v^{*}$ is a solution of problem (1) we have

$$
\bar{L}\left(v^{*}\right)=\sup _{l \in\left(L_{2}(\Gamma)\right)^{+}}\left\{J\left(v^{*}\right)-\int_{\Gamma} l \gamma v^{*} d \Gamma\right\}=J\left(v^{*}\right)=\chi(0) .
$$

Furthermore, we have

$$
\begin{aligned}
\underline{L}\left(l^{*}\right) & =\inf _{v \in W_{2}^{1}(\Omega)} L\left(v, l^{*}\right) \\
& =\inf _{v \in W_{2}^{1}(\Omega)} \inf _{m \in L_{2}(\Gamma)} \overline{\mathrm{K}}\left(v, l^{*}, m\right) \\
& =\inf _{m \in L_{2}(\Gamma)} \inf _{v \in W_{2}^{1}(\Omega)} \overline{\mathrm{K}}\left(v, l^{*}, m\right) \\
& =\inf _{m-\gamma v \leq m} \inf _{v \in W_{2}^{1}(\Omega)}\left\{J\left(v^{*}\right)+\int_{\Gamma} l^{*} m d \Gamma\right\} \\
& =\inf _{m \in L_{2}(\Gamma)}\left\{\chi(m)+\int_{\Gamma} l^{*} m d \Gamma\right\} .
\end{aligned}
$$

Hence, we get

or

$$
\chi(0)=\inf _{m \in L_{2}(\Gamma)}\left\{\chi(m)+\int_{\Gamma} l^{*} m d \Gamma\right\},
$$

Since

$$
\chi(m)+\int_{\Gamma} l^{*} m d \Gamma \geq \chi(0) \quad \forall m \in L_{2}(\Gamma) .
$$

$$
\chi(m)+\int_{\Gamma} l^{*} m d \Gamma+\frac{r}{2} \int_{\Gamma} m^{2} d \Gamma \geq \chi(m)+\int_{\Gamma} l^{*} m d \Gamma \geq \chi(0),
$$


it follows from Theorem 2.3 that $\left(v^{*}, l^{*}\right)$ is a saddle point of $M(v, l)$.

Theorem 2.5. Let $\left(v^{*}, l^{*}\right)$ be a saddle point of the modified Lagrangian functional $M(v, l)$ in $(6)$. Then $\left(v^{*}, l^{*}\right)$ is a saddle point of the classical Lagrangian functional $L(v, l)$ given in (4).

Proof. Let us show that $-l^{*} \in \partial \chi(0)$, where $\partial \chi(0)$ is the subdifferential of the convex function $\chi(m)$ at the point zero, which means

$$
\chi(m)-\chi(0) \geq-\int_{\Gamma} l^{*} m d \Gamma \quad \forall m \in L_{2}(\Gamma) .
$$

We will give the proof via a contradiction. Suppose that we had $-l^{*} \notin \partial \chi(0)$. Then there exists an $\bar{m} \in L_{2}(\Gamma)$ such that

$$
\chi(\bar{m})+\int_{\Gamma} l^{*} \bar{m} d \Gamma<\chi(0) .
$$

Let $\zeta=\chi(0)-\chi(\bar{m})-\int_{\Gamma} l^{*} \bar{m} d \Gamma>0$ and let $m(\lambda)=\lambda \bar{m}+(1-\lambda) \cdot 0, \forall \lambda \in(0,1)$. From Theorem 2.3, we have

$$
\chi(m)+\int_{\Gamma} l^{*} m d \Gamma+\frac{r}{2} \int_{\Gamma} m^{2} d \Gamma \geq \chi(0) \quad \forall m \in L_{2}(\Gamma) .
$$

Therefore, if $m=\lambda \bar{m}$, we get

$$
\begin{aligned}
\chi(0) & \leq \chi(m(\lambda))+\lambda \int_{\Gamma} l^{*} \bar{m} d \Gamma+\frac{r \lambda^{2}}{2} \int_{\Gamma} \bar{m}^{2} d \Gamma \\
& \leq \lambda \chi(\bar{m})+(1-\lambda) \chi(0)+\lambda \int_{\Gamma} l^{*} \bar{m} d \Gamma+\frac{r \lambda^{2}}{2} \int_{\Gamma} \bar{m}^{2} d \Gamma \\
& =\lambda\left(\chi(\bar{m})-\chi(0)+\int_{\Gamma} l^{*} \bar{m} d \Gamma\right)+\chi(0)+\frac{r \lambda^{2}}{2} \int_{\Gamma} \bar{m}^{2} d \Gamma,
\end{aligned}
$$

and

$$
\lambda\left(\chi(0)-\chi(\bar{m})-\int_{\Gamma} l^{*} \bar{m} d \Gamma\right) \leq \frac{r \lambda^{2}}{2} \int_{\Gamma} \bar{m}^{2} d \Gamma
$$

as well as

$$
0<\zeta=\chi(0)-\chi(\bar{m})-\int_{\Gamma} l^{*} \bar{m} d \Gamma \leq \frac{r \lambda}{2} \int_{\Gamma} \bar{m}^{2} d \Gamma .
$$

By letting $\lambda=0$ in the last inequality set, we obtain the contradiction $0<0$. Hence, we must have $-l^{*} \in \partial \chi(0)$, which implies

$$
\chi(m)+\int_{\Gamma} l^{*} m d \Gamma \geq \chi(0) \quad \forall m \in L_{2}(\Gamma) .
$$

It follows from (20) that we get

$$
\int_{\Gamma} l^{*} m d \Gamma \geq \chi(0)-\chi(m) \geq 0 \quad \forall m \in\left(L_{2}(\Gamma)\right)^{+},
$$


and, hence, $l^{*} \in\left(L_{2}(\Gamma)\right)^{+}$. We then have

$$
\underline{L}\left(l^{*}\right)=\inf _{v \in W_{2}^{1}(\Omega)} L\left(v, l^{*}\right)=\inf _{v \in W_{2}^{1}(\Omega)}\left\{\chi(m)+\int_{\Gamma} l^{*} m d \Gamma\right\} .
$$

From (20), we get

$$
\underline{L}\left(l^{*}\right) \geq \chi(0) .
$$

Furthermore, taking into account that $v^{*}$ is a solution of problem (1), we have

$$
\begin{aligned}
\bar{L}\left(v^{*}\right) & =\sup _{l \in\left(L_{2}(\Gamma)\right)^{+}} L\left(v^{*}, l\right)=\sup _{l \in\left(L_{2}(\Gamma)\right)^{+}}\left\{J\left(v^{*}\right)-\int_{\Gamma} l \gamma v^{*} d \Gamma\right\} \\
& =J\left(v^{*}\right)=\chi(0) .
\end{aligned}
$$

Therefore, we obtain

$$
\chi(0)=\bar{L}\left(v^{*}\right) \geq \underline{L}\left(l^{*}\right) \geq \chi(0)
$$

that is,

$$
\bar{L}\left(v^{*}\right)=\underline{L}\left(l^{*}\right),
$$

which implies that $\left(v^{*}, l^{*}\right)$ is a saddle point of $L(v, l)$.

\section{Conclusion and discussion}

As we mentioned, other well-known methods based on classical Lagrangian functionals do not guarantee the convergence to a saddle point in semicoercive variational inequality. Theorems 2.4 and 2.5 allow to consider corresponding methods based on modified Lagrangian functionals. For example, the Uzawa method in [1] can be used, and the algorithm can be described as follows:

Choose an arbitrary initial point $l^{0} \in W_{2}^{1 / 2}(\Gamma)$, that is the trace on $\Gamma$ of a function $u^{0} \in W_{2}^{1}(\Omega)$.

U-Step1: at the $k$ th $\operatorname{step}(k=0,1, \ldots)$, set

$$
u^{k+1}=\arg \min _{v \in W_{2}^{1}(\Omega)} M\left(v, l^{k}\right) .
$$

U-Step2: find $l^{k+1}$ by the formula

$$
l^{k+1}=\left(l^{k}-r \gamma u^{k+1}\right)^{+} .
$$

According to Theorem 2.1, the points $u^{k}(k=1,2, \ldots)$ defined in (21) all exist. They are defined uniquely if $u^{k} \in W_{2}^{2}(\Omega)$ (cf. [1]). However, to find the element $u^{k+1}$ at U-Step 1 of the Uzawa algorithm, one must minimize the functional $M\left(v, l^{k}\right)$, which is not strongly convex with respect to the variable $v$.

Woo et al. [8] introduced a method for solving problem (1) based on the combination of the modified Lagrangian functional with the proximal regularization. That algorithm can be summarized as follows:

Given an arbitrary starting point $\left(u^{0}, l^{0}\right) \in W_{2}^{1}(\Omega) \times W_{2}^{1 / 2}(\Gamma)$, the sequence $\left\{\left(u^{k}, l^{k}\right)\right\}$ is generated by 
W-Step1: at the $k$ th step $(k=0,1, \ldots)$, the functional

$$
L_{k}(v)=M\left(v, l^{k}\right)+\frac{1}{2}\left\|v-u^{k}\right\|_{L_{2}(\Gamma)}^{2} \quad \forall v \in W_{2}^{1}(\Omega)
$$

is constructed and the point

$$
u^{k+1}=\arg \min _{v \in W_{2}^{1}(\Omega)} L_{k}(v)
$$

is determined.

W-Step2: the dual variable $l^{k+1}$ is corrected by the formula

$$
l^{k+1}=\left(l^{k}-r \gamma u^{k+1}\right)^{+} .
$$

The regularizing addition $\frac{1}{2}\left\|v-u^{k}\right\|_{L_{2}(\Omega)}^{2}$ in W-Step 1 provides the strong convexity of the minimized functionals $L_{k}(v)$. This guarantees that each of the auxiliary problems

$$
\left\{\begin{array}{l}
L_{k}(v)-\min \\
v \in W_{2}^{1}(\Omega)
\end{array}\right.
$$

has a unique solution, which can be found by efficient optimization methods.

Under the assumptions

- $u^{k} \in W_{2}^{2}(\Omega), k=1,2, \ldots$,

- $\left\|u^{k}\right\|_{W_{2}^{2}(\Omega)} \leq C$, where $C>0$ is a constant,

one can show that the uniqueness of the saddle point of the Lagrangian functional $L(v, l)$ or $M(v, l)$ and the convergence in $W_{2}^{1}(\Omega) \times L_{2}(\Gamma)$ to a unique saddle point of the sequence $\left\{\left(u^{k}, l^{k}\right)\right\}$ generated by the W-Steps 1 and 2 .

The method of Woo et al. combined with the finite element method was introduced in [7].

\section{References}

[1] G. Duvaut and J. L. Lions, Inequalities in Mechanics and Physics, Springer-Verlag, Berlin, 1976; Nauka, Moscow, 1980

[2] G. Fikera, Existence Theorems in Elasticity, Springer-Verlag, Berlin, 1976; Nauka, Moskow, 1980

[3] I. Glavaček, J. Haslinger, I. Nečas, and J. Lovišek, Numerical Solution of Variational Inequalities, Springer-Verlag, Berlin, 1988; Mir, Moscow, 1986.

[4] R. Glowinski, Numerical Methods for Nonlinear Variational Problems, Springer-Verlag, New York, 1984

[5] R. Glowinski, J. L. Lions, and R. Tremolieres, Numerical Analysis of Variational Inequalities, North-Holland, Amsterdam, 1981.

[6] N. Kikuchi and T. Oden, Contact Problem in Elasticity: A Study of Variational Inequalities and Finite Element Methods, SIAM, Philadelphia, 1988.

[7] R. V. Namm and S. A. Sachkov, Solution of the quasi-variational Signorini inequality by the method of successive approximations, Zh. Vychisl. Mat. Mat. Fiz. 49 (2009), no. 5, 805-814; translation in Comput. Math. Math. Phys. 49 (2009), no. 5, 776-785.

[8] G. Vu, S. Kim, R. V. Namm, and S. A. Sachkov, The method of iterative proximal regularization for finding a saddle point in the semi-coercive Signorini problem, Zh. Vychisl. Mat. Mat. Fiz. 46 (2006), no. 11, 2024-2031; translation in Comput. Math. Math. Phys. 46 (2006), no. 11, 1932-1939. 
[9] G. Woo, R. V. Namm, and S. A. Sachkoff, An iterative method, based on a modified Lagrange functional, for finding a saddle point for the semi-coercive Signorini problem, Zh. Vychisl. Mat. Mat. Fiz. 46 (2006), no. 1, 26-36; translation in Comput. Math. Math. Phys. 46 (2006), no. 1, 23-33.

RoBert V. NAMM

Pacific National University

TikHookeanskaya ST., 136, Khabarovsk

680035 RUSSIA

E-mail address: namm@mail.khstu.ru

Gyungsoo Woo

Department of Mathematics

Changwon National University

Changwon 641-773, Korea

E-mail address: gswoo@changwon.ac.kr

Shu-Sen XIe

School of Mathematical Sciences

OCEAN University of China

QINGDAO 266071, P. R. CHINA

E-mail address: shusenxie@ouc.edu.cn

Sucheol Yi

Department of Mathematics

Changwon National University

Changwon 641-773, Korea

E-mail address: scyi@changwon.ac.kr 